\title{
Aux sources du monachisme martinien, Les Vies de Martin en prose et en vers
}

At the source of Martinian monasticism, the lives of saint Martin in prose and verses

\section{Sylvie Labarre}

\section{(2) OpenEdition}

\section{Journals}

\section{Édition électronique}

URL : http://journals.openedition.org/abpo/2472

DOI : $10.4000 / a b p o .2472$

ISBN : 978-2-7535-2136-0

ISSN : 2108-6443

\section{Éditeur}

Presses universitaires de Rennes

\section{Édition imprimée}

Date de publication : 30 octobre 2012

Pagination : $27-40$

ISBN : 978-2-7535-2134-6

ISSN : 0399-0826

Référence électronique

Sylvie Labarre, "Aux sources du monachisme martinien, Les Vies de Martin en prose et en vers », Annales de Bretagne et des Pays de l'Ouest [En ligne], 119-3 | 2012, mis en ligne le 30 octobre 2014, consulté le 20 avril 2019. URL : http://journals.openedition.org/abpo/2472 ; DOI : 10.4000/abpo.2472 


\title{
Aux sources du monachisme martinien, les Vies de Martin en prose et en vers
}

\author{
Sylvie LABARRE \\ Maître de conférences en langue et littérature latines, Université du Maine \\ Institut d'Études Augustiniennes, UMR 8584
}

La Vie de saint Martin, rédigée par Sulpice Sévère en 396-397, ne compte pas à proprement parler parmi les Vies de moines, comme la Vie d'Antoine par Athanase (vers 356), les Vies de moines de Jérôme (entre 376 et 392) ou l'Histoire des moines d'Égypte traduite en latin par Rufin (vers 404). Les manuscrits médiévaux de celle-ci portent d'ailleurs le plus souvent pour titre Vita sancti Martini episcopi, sans jamais mentionner le moine. Martin n'a pas non plus rédigé de règle monastique, alors qu'on en dénombre près d'une trentaine en langue latine à l'époque ancienne ${ }^{1}$; du reste le terme " règle ", au sens technique, n'apparaîtra que plus tard. Cependant on relève trente-quatre occurrences du mot monachus et quarante du mot monasterium dans la Vie de saint Martin, les trois Lettres et les Dialogues de Sulpice ${ }^{2}$. Le monachisme est bien présent dans ces œuvres, mais sans en constituer le thème essentiel.

Après plusieurs tentatives de vie érémitique, Martin fonde un monastère à Ligugé, qui demeure le plus ancien de France, et un autre à Marmoutier. Grâce à sa personnalité charismatique et au talent de son biographe, il étend en outre son influence spirituelle au-delà des monastères qu'il a luimême fondés. D'importants travaux ont été consacrés, notamment entre 1994 et 2003, au monachisme ancien, faisant une place au monachisme martinien, par Adalbert de Vogüé et Vincent Desprez, en France, cependant qu'en Allemagne, les ouvrages de Maria-Elisabeth Brunert, sur L'idéal de l'ascétisme au désert, et d'Eva Elm, sur Le pouvoir de la sagesse, apportaient un éclairage très intéressant sur la figure de l'évêque et du moine ${ }^{3}$.

1. Desprez, Vincent, Règles monastiques d'Occident, $I V^{e}-V^{e}$ siècle. D'Augustin à Ferréol, Bellefontaine, 1980, p. 19-25.

2. SulPICE SÉvĖre, Vie de saint Martin, intro., éd., trad., com. de Jacques FonTAINE, SC 133-135, Paris 1967-69; Gallus. Dialogues sur les « vertus » de saint Martin, intro., éd., trad., com. de Jacques FonTAINE, SC 510, Paris, 2006.

3. VoGüÉ, Adalbert de, Histoire littéraire du mouvement monastique dans l'Antiquité, IV, 1 : Le monachisme latin, Paris, 1997, p. 19-156; Regards sur le monachisme des pre- 
Pour ma part, je me suis fixé pour projet de relire les textes en prose de Sulpice Sévère, la Vita Martini et les Dialogues, afin d'y relever les éléments d'une regula non écrite, qui serait à l'origine du monachisme martinien, puis de compléter cet inventaire par les caractères principaux de l'évêquemoine, selon Sulpice, pour ensuite examiner, à travers les réécritures de Paulin de Périgueux et Venance Fortunat ${ }^{4}$, quel regard les poètes ont porté sur le monachisme martinien, aux siècles suivants.

\section{Les principaux thèmes monastiques dans la Vie et les Dialogues de Sulpice Sévère}

Notre source la plus ancienne est constituée par les écrits en prose de Sulpice Sévère, un avocat aquitain né vers 360, ami de Paulin de Nole, contemporain et admirateur de Martin, converti à l'ascétisme chrétien. Les passages les plus importants pour le monachisme se situent, avant l'épiscopat, avec la fondation de Ligugé, après l'épiscopat, avec la fondation de Marmoutier, puis dans le portrait final, qui clôt la Vie. Des éléments sont également disséminés à travers les Dialogues. Les principaux thèmes relevés par les spécialistes du monachisme ${ }^{5}$ s'y trouvent abordés.

\section{La séparation du monde}

L'aspiration au désert est présentée par Sulpice comme une volonté constante de Martin dès son jeune âge (Vie de saint Martin, 2, 4 : " à douze ans, il désira vivre au désert "). L'hagiographie offre en effet une lecture unifiée de la vie de son héros et insiste sur la permanence de ses qualités et de ses desseins ${ }^{6}$. La première expérience se situe à Milan (V. M., 6, 4 : sibi monasterium statuit, " il s'installa un ermitage "). Il se retire ensuite dans l'île de Gallinara $(V . M ., 6,5)$, avant de " s'installer un ermitage " à Ligugé (V.M., 7, 1 : haut longe sibi ab oppido monasterium conlocauit). La formule employée pour Milan est reprise pour la fondation de Marmoutier : duobus fere extra ciuitatem milibus monasterium sibi statuit (V.M., 10, 3). Dans tous ces passages, monasterium a le sens étymologique de « habitation de moine

miers siècles : recueil d'articles, Studia Anselmania, 130, Roma, 2000; DESPREZ, Vincent, Le monachisme primitif des origines jusqu'au concile d'Éphèse, Bellefontaine, 1998, ch. 14 "Martin moine, fondateur, évêque ", p. 547-561; BRUNERT, Maria-Elisabeth, Das Ideal der Wüstenaskese und seine Rezeption in Gallien bis zum Ende des 6. Jahrhunderts, Münster, 1994, p. 145-176; Elm, Eva, Die Macht der Weisheit : das Bild des Bischofs in der Vita Augustini des Possidius und anderen spätantiken und frühmittelalterlichen Bischofsviten, Leiden, 2003, p. 78-90.

4. LABARRE, Sylvie, Le Manteau partagé : deux métamorphoses poétiques de la Vie de saint Martin chez Paulin de Périgueux (Ve siècle) et Venance Fortunat ( Vee siècle), Collection des Études Augustiniennes, série Antiquité, 158, Paris, 1998.

5. Voir de VoGüÉ, Adalbert, "Pour lire les anciens moines ", dans Regards sur le monachisme..., p. 3-27.

6. LABARRE, Sylvie, "La composition de la Vita Martini de Sulpice Sévère ", Vita Latina 171 (décembre 2004), p. 102-120. 
seul ", donc " ermitage " ou " cellule ", comme chez Jérôme (Vie de Paul, 12, 4) ou Égérie, dans son Journal de voyage, à maintes reprises (par ex. 16, 2).

L'ermitage de Martin n'est jamais loin d'une ville. Pourtant Sulpice décrit Marmoutier, situé à trois kilomètres du castrum de Tours, de l'autre côté du fleuve, comme un désert ${ }^{7}$ : " cette retraite était si écartée qu'elle n'avait rien à envier à la solitude d'un désert. D'un côté, en effet, elle était entourée par la falaise à pic d'un mont élevé, et le reste du terrain était enfermé dans un léger méandre de la Loire " $(10,4)$. On songe à la haute Thébaïde d'Antoine, car l'auteur " projette " " la topique du paysage ascétique oriental " sur " une solitude monastique d'Occident ${ }^{8}$ ".

Ni à Ligugé, ni à Marmoutier, Martin n'observe cependant de " clôture stricte ", puisque c'est à l'occasion de ses sorties qu'il opère des miracles (ex. V.M., 8, 1), ou bien encore, bien qu'il soit " difficile de le tirer de son monastère " (cum erui monasterio suo non facile posset), il accepte de le quitter, quand on vient lui demander secours (ex. V.M., 9, 1). Ainsi faisait Antoine, mais il soulignait lui-même combien la solitude était essentielle au moine, en recourant à la fameuse comparaison du moine dans sa retraite et du poisson dans l'eau : "Comme les poissons meurent lorsqu'ils restent longtemps sur la terre sèche, de même à s'attarder avec vous et à séjourner chez vous, les moines se relâchent. Il faut donc que, comme le poisson vers la mer, nous nous hâtions vers la montagne, pour ne pas oublier, en nous attardant, les choses intérieures ${ }^{9}$."

\section{La vie en communauté}

Dans l'armée, Martin inspirait déjà à ses camarades affection et respect, en faisant preuve à leur égard de charité, de patience et d'humilité (V.M., 2,7 ), et des liens de camaraderie le liaient si fortement à son tribun qu'il attendit pour quitter la carrière militaire le moment où cet homme renoncerait lui-même au monde. Ces périodes de vie commune préfigurent les relations communautaires des moines. À Ligugé, un catéchumène se joint à lui. À Gallinara, il est accompagné par un prêtre $(6,5)$. À Marmoutier, ce sont environ quatre-vingts disciples qui se joignent à lui. Ils sont souvent issus de l'aristocratie (V.M., 2, 7 : multi inter eos nobiles habebantur). Cela correspond bien à ce que nous savons du recrutement ecclésiastique, en Gaule, à la fin de l'Antiquité ${ }^{10}$. Cependant Sulpice signale la présence de deux mille moines, venus pour la plupart de la Touraine, lors des funérailles

7. Article " érémitisme ", Dictionnaire de spiritualité, t. 4, Paris, 1960, col. 936-982.

8. Fontaine, Jacques, SC 134, p. 667. Athanase, Vie d'Antoine, SC 400, 12, 3.

9. Athanase, Vie d'Antoine, 85, 1-5.

10. Heinzelmann, Martin, Bischofsherrschaft in Gallien. Zur Kontinuät römischer Führungsschichten vom 4. bis zum 7. Jahrhundert. Soziale, prosopographische und bildungsgeschichtliche Aspekte, Beihefte der Francia, Bd. 5, München, 1976; Consolino, Franca Ela, Ascesi e mondanità nella Gallia tardoantica. Studi sulla figura del vescovo nei secoli IV-VI, Koinonia 4, Napoli, 1979. 
du saint en 397; ce qui laisse supposer que le recrutement s'étaitconsidérablement élargi.

Pour la description de la " colonie monastique de Marmoutier " et son organisation, on se reportera à l'ouvrage fondamental de Luce Pietri : $L a$ Ville de Tours du IV au VIe siècle. Naissance d'une cité chrétienne, Rome, 1983, p. 421-430 et 652 sq. Le monasterium primitif n'est pas un monastère au sens courant du mot, mais en fait un " groupement d'ermitages ${ }^{11}$ ", dispersés sur une assez vaste superficie entourée d'une enceinte. On voit même un moine appelé Clair s'installer à quelque distance du " monastère épiscopal ", hors " clôture " (V.M., 23, 2). L'autorité de Martin peut même y être contestée. Ainsi Brice, son futur successeur à l'épiscopat, entré très tôt dans la communauté et éduqué par le saint, l'insulte (Dial., III, 15).

\section{L'ascèse}

Chaque moine s'exerce à une ascèse individuelle, dont témoigne le type d'habitat choisi. Selon Sulpice, Martin habite une " cellule construite en bois " $(10,4)$. Selon une inscription métrique transmise par les manuscrits martiniens, appelés "Martinelli ", il s'agirait cependant d'une grotte : "Ici habita le bienheureux ermite dans la grotte ${ }^{12}$. " Une autre décrit son lit comme une banquette de pierre recouverte de cendres : "Un cilice, une pierre sous la tête et de la cendre épaisse, pour tes membres fatigués, c'était là, à tes yeux, un lit. Car cette banquette servait à ton repos dans la nuit silencieuse; en guise de siège ou de chaire, le jour, ce banc aussi ${ }^{13}$." Les autres moines occupent des cabanes en bois ou creusent dans la falaise de craie des habitats troglodytiques (V.M., 10,5). Les seuls locaux communs sont l'oratoire, où les moines se réunissent pour les offices, et le réfectoire, où ils partagent leur repas.

La nourriture est tout à fait frugale. À Gallinara, Martin vivait de « racines de plantes " (V.M., 6, 5 : radicibus uixit herbarum), comme les anachorètes d'Égypte, à propos desquels Sulpice emploie exactement la même expression, dans les Dialogues (Dial., I, 15, 2; 16, 1). À Marmoutier, les moines pratiquent le jeûne dans leur cellule, avant de se retrouver pour un repas commun, dont le vin est exclu, sauf pour les malades (V.M., 10, 7).

Toute propriété est exclue, parce que chacun renonce à ses biens (V.M., $10,6)$ et que le travail artisanal y est interdit. En cela, le monachisme mar-

11. FonTAINE, Jacques, SC 134, p. 679.

12. LE BLANT, Edmond, Inscriptions chrétiennes de la Gaule antérieures au VIII siècle, t. 1, Paris, 1856, n ${ }^{\circ} 167$, v. 7 : "Hic inhabitauit felix eremita sub antro. " DESPREZ, Vincent, Le Monachisme primitif des origines jusqu'au concile d'Éphèse, Bellefontaine, 1998, p. 555. PIETRI, Luce, op. cit., Appendice 6, p. 802-804. Voir aussi GILARDI, F. J., The Sylloge Epigraphica Turonensis de S. Martino, diss. Washington, D. C., 1983 (compte rendu critique de Luce Pietri dans Francia, 12 (1984), p. 621-631).

13. LE BLANT, n 169, v. 5-8: "Cilicium, subter capiti lapis et cinis altus / Exesis membris hic, uidebare, torus./Sellula namque fuit requies in nocte silenti, / Pro scamno aut cathedra huic haec quoque sella die." 
tinien se différencie des communautés régies par les Règles occidentales et astreintes au travail manuel : Augustin écrit un De opere monachorum. Ainsi le monastère salarie un paysan, pour le transport du bois (V.M., 21, 3). Le seul travail permis est la copie des manuscrits par les plus jeunes (V.M., 10,6). La subsistance de la communauté martinienne doit donc être assurée par les dons privés et les subventions de l'Église de Tours. Quand Lycontius fait une riche offrande à Martin, les moines en réclament une partie pour leur nourriture et leurs vêtements (Dial., III, 14, 6).

\section{Le vêtement}

Le vêtement du moine fait l'objet de recommandations qui figurent en bonne place dans les Règles monastiques, par exemple au début du Practicos d'Évagre (Prologue) ou des Institutions cénobitiques de Cassien (Livre premier). En effet, il marque la séparation du monde et la vie commune. Or on peut imaginer chez les premiers moines de Marmoutier la même disparité dans le vêtement que dans l'habitat. D'après Sulpice, " bon nombre s'habillaient de poil de chameau " (V.M., 10, 8). Martin se signale, quant à lui, par une tenue inconvenante aux yeux de la hiérarchie ecclésiastique. Mais son biographe explique que l'humilité de son cœur se traduit par la pauvreté de son vêtement (V.M., 10, 2: "Même humilité en son cœur, même pauvreté dans son vêtement "). Le saint porte également un cilice pour sa mortification (V.M., 14, 4).

\section{La prière}

Elle apparaît comme le point capital de la doctrine monastique. En effet, le moine réalise le précepte évangélique et apostolique : "Priez sans cesse " (Lc 18, 1; 1 Thess. 5, 16). Si les moines ne peuvent travailler de leurs mains, c'est qu'ils sont entièrement adonnés à la prière. Tandis que les plus jeunes copiaient des manuscrits, "leurs aînés vaquaient à la prière " (V.M., 10, 6). La prière individuelle n'est interrompue que par la prière collective : " on ne sortait que rarement de sa cellule, sauf pour se réunir au lieu de la prière " (V.M., 10, 7). Elle peut également se traduire par la récitation de psaumes ou le chant d'hymnes et de psaumes (V.M., 23, 8 et 9). Là encore, on songe à la Vie d'Antoine : "Dans les montagnes, les ermitages étaient donc comme des tentes remplies de chœurs divins, chantant des psaumes, lisant les Écritures, jeûnant, priant, se réjouissant dans l'espérance des biens futurs, travaillant pour faire l'aumône et menant une vie d'amour mutuel et de concorde" $(44,2)$.

\section{La séparation des sexes et l'exaltation de la virginité}

Certains récits, rapportés par Gallus, au livre II des Dialogues, nous éclairent sur la volonté de Martin de maintenir séparés les hommes et les femmes consacrés. Il approuve cette vierge qui refuse sa visite (II, 12, 
1-11), et persuade un soldat devenu moine de ne pas cohabiter avec sa femme, elle-même moniale (II, 11). Une parabole, développée juste avant, indique clairement que la virginité est tenue supérieure au mariage (II, 10). Elle se conclut ainsi : "Il n'est rien de comparable à la virginité. Ainsi, ceux qui assimilent le mariage à la fornication sont dans une grave erreur, tout comme ceux qui estiment que le mariage doit être égalé à la virginité, sont de bien misérables fous. " Martin s'oppose donc à la thèse du moine Jovinien qu'Ambroise, Sirice et Jérôme, dans son Aduersus Iouinianum, ont également condamnée, selon laquelle le mariage pouvait être considéré l'égal de la virginité.

Malgré la présence de ces thèmes monastiques, le propos de Sulpice Sévère est moins de faire un tableau du monachisme martinien que de tracer le portrait d'un personnage tout à fait unique et qui a suscité de vives critiques de la part de certains évêques (V.M., 27, 3).

\section{Le portrait de l'évêque-moine}

Martin possède les qualités monastiques que sont la charité et l'humilité. Sa sobriété (frugalitas) est signalée, alors qu'il sert encore dans l'armée (V.M., 2, 7 : " on l'aurait cru moine et non pas soldat "). Mais des traits plus originaux se dessinent. Sulpice représente un moine tourné vers l'action : Martin entreprend des expéditions contre l'hérésie arienne en Illyrie (V.M., 6 , 4). Son enseignement se fait par l'exemple et non par la parole (V.M., 10, 5) : "Ils se formaient à l'exemple de leur bienheureux maître. "

Le thème de la prière, véritable leitmotiv de la Vie de saint Martin, est repris dans le portrait final du saint : "Selon l'habitude des forgerons qui frappent sur leur enclume dans l'intervalle de leur travail, en quelque sorte pour alléger leur peine, ainsi Martin priait sans cesse, même quand il avait l'air de faire autre chose ${ }^{14}$. "Au livre de l'Ecclésiastique, Ben Sira décrit le forgeron qui passe ses veilles à parfaire son ouvrage (Eccli., 38, 29). En maints passages, Martin est représenté en prière; c'est d'elle qu'il tire sa uirtus agissante (par ex. pour la résurrection d'un catéchumène, Vie de saint Martin, 7, 3 : « il attendait avec une confiance absolue l'effet de sa prière et de la miséricorde du Seigneur "; pour la destruction d'un temple à Levroux, 14, 4 : « Là pendant trois jours, vêtu d'un cilice et couvert de cendre, dans le jeûne et l'oraison ininterrompus, il adressait sa prière au Seigneur, afin que la vertu divine renversât ce temple "). Or Martin disait, d'après Sulpice, avoir eu des pouvoirs thaumaturgiques supérieurs, quand il n'était qu'un simple moine (Dial., II, 4, 1-3).

Martin pratique l'idéal antique de la vie mixte qui allie action et méditation, solitude et présence au monde. Cet idéal s'épanouit dans la double condition d'évêque et de moine. Le chapitre consacré à Marmoutier com-

14. SulPICE SÉVÈRE, Vie de saint Martin, 26, 4 : " ut fabris ferrariis moris est, qui inter operandum pro quodam laboris leuamine incudem suam feriunt, ita Martinus, etiam dum aliud agere uideretur, semper orabat." 
mence par l'alliance de la dignité de l'évêque et de la vertu du moine que Martin réalise $(10,2)$. Il se clôt sur la formation dans son monastère de futurs évêques : "Nous en avons vu plusieurs (moines) devenir ensuite évêques. Quelle était en effet la cité ou l'église qui n'aurait point désiré un pontife sorti du monastère de Martin?" (10, 8-9).

Martin partage la double qualité de moine et d'évêque avec Héliodore d'Altinum ${ }^{15}$, correspondant de Jérôme, qui devint prêtre, après une carrière militaire, et participa au concile d'Aquilée en 381, ou Eusèbe, élu à la chaire épiscopale de Verceil en $345^{16}$, ou bien encore Alypius, auquel Paulin de Nole adresse une lettre et qu'il loue de s'être fait pour lui-même un désert ${ }^{17}$. Toutefois il demeure pour ses contemporains un évêque-moine tout à fait atypique. Au cours des siècles suivants, la polémique s'estompe et le culte de Martin triomphe.

\section{Quel regard est porté sur le monachisme martinien dans les Vies poétiques des $\mathrm{V}^{\mathrm{e}}$ et $\mathrm{VI}^{\mathrm{e}} \mathrm{s}$ iècles?}

\section{Paulin de Périgueux : une réécriture plus occidentale, plus actuelle et plus tourangelle}

Paulin de Périgueux compose une longue épopée hagiographique ${ }^{18} \mathrm{de}$ 3622 hexamètres, qui met en vers les deux œuvres de Sulpice Sévère dont nous avons parlé, en suivant scrupuleusement l'ordre de son récit. Nous savons peu de choses de lui, si ce n'est qu'il est gaulois et peut-être évêque. $\mathrm{Au} \mathrm{VI}^{\mathrm{e}}$ siècle, il était confondu avec Paulin de Nole par Grégoire de Tours (De uirtutibus s. Martini, I, 2) et Venance Fortunat. C'est la lettre de dédicace par laquelle il adresse son œuvre à l'évêque de Tours, Perpetuus (458/9-488/9), qui nous permet de le situer dans le temps et de voir en lui un des artisans du culte de Martin. Comment aborde-t-il le monachisme martinien?

Il emploie quinze fois monachus ${ }^{19}$. La plupart des occurrences concernent les moines qui entourent Martin. Chaque fois que monachus se rapporte à Martin, c'est dans un contexte de louange, non pas pour dire simplement que Martin est moine, mais pour exprimer les qualités cumulatives

15. JÉRÔME, Lettres, éd. J. LABOURT, 60, 10 (lettre à Héliodore, éloge funèbre de son neveu Népotien) : "In uno atque eodem et imitabatur monachum et episcopum uenerabatur."

16. AmBroise, Lettres, 63, 66, éd. FALLER, CSEL 82, P 10, 3, p. 270 : " monasterii continentia et disciplina ecclesiae."

17. PAULIN DE Nole, Lettres, éd. HARTEL, CSEL 29, 3, 2 : "nam etsi in populis ac super populum agas oues pascuae domini regens sollicitis uigil pastor excubiis, tamen abdicatione saeculi et repulsa carnis ac sanguinis desertum tibi ipse fecisti secretus a multis, uocatus in paucis."

18. L'édition de référence est celle de M. Petschenig (CSEL 16, 1, Vienne, 1888). Mais je cite ma propre édition et ma traduction à paraître dans la collection "Sources chrétiennes".

19. PAulin de PÉRIgueuX, Vie de saint Martin, I, 135; II, 12, 84, 95, 299, 321 ; III, 148, 172, 185 $274 ;$ IV, 42, 596, 608; V, 549, 684. 
qu'il réunit en lui : il est à la fois moine et soldat, moine et évêque et en outre un maître ${ }^{20}$.

En revanche, il n'emploie pas une seule fois monasterium, sans doute à cause de la difficulté métrique (une brève, deux longues, une brève), qui n'arrête pourtant nullement Fortunat ${ }^{21}$. Il lui préfère cella : I, 257 (Milan); 296 (Ligugé); II, 90 (Marmoutier); VI, 74 (Marmoutier), qui traduit bien le caractère individuel de l'habitat et insiste sur la solitude du moine.

Paulin décrit avec une grande précision le site de Marmoutier : " D’un côté, la paroi rocheuse, incurvée, présentant un léger renfoncement, l'entourait d'un rebord formé par l'escarpement naturel du rocher, de l'autre, la Loire, grâce aux faibles sinuosités de ses rives recourbées, isolait cette langue de terre par une avancée d'eau. Un seul accès étroit s'ouvrait entre la paroi rocheuse et le fleuve, là où le courant, tout en restant un peu à l'écart et en ménageant un sentier resserré, frôle les bords de la falaise ${ }^{22}$." Le poète apporte des détails supplémentaires à la description de l'étroite plaine alluviale ménagée par un méandre de la Loire, entre le cours du fleuve et le flanc abrupt du coteau. Est-ce la traduction d'une connaissance personnelle de ce lieu ou bien l'effet de l'amplification poétique? Sans doute les deux à la fois.

En outre, ce passage comporte des réminiscences littéraires, plus ou moins évidentes. Paulin rapproche, par le réemploi de praecisi... saxi, expression de l'Énéide, la retraite de Martin et l'antre de Cacus ${ }^{23}$. De manière surprenante, le locus horridus où vit le monstre Cacus, qui ravage l'Arcadie et occupe un espace hors de l'humanité, est transformé en un locus " édifiant ", où le saint en prière rencontre Dieu. Or nous avons pu observer que Paulin de Périgueux avait une connaissance très fine de l'épopée virgilienne et un souvenir très précis du contexte dans lequel une expression avait été employée par le poète de Mantoue ${ }^{24}$. Ce renversement nous semble tout à fait conscient : il montre comment la foi en Dieu met de l'ordre dans le chaos.

20. PAUlin DE PÉRIgUeux, Vie de saint Martin, I, 135 : "iam meritis monachus, sed solo nomine miles "; II, 12 : " hactenus, ut mores monachi uel gesta referrem "; II, 84 : " nec minor inde labor monachi, quia gloria creuit »; II, 95 : " secretum hospitium monacho, uia parua magistro." "

21. Fortunat scande en effet la syllabe -te comme une brève. Voir I, 158: «Fitque monasterium Pictaua cominus urbe " ; I, 220 : "inde monasterium sibi condit rupe sub alta" ; IV, 158, 391, 483. Voir L. QuicherAT, Thesaurus poeticus linguae latinae, Paris, 1890, art. " monasterium ", p. 690.

22. PAulin DE PÉRIGUEuX, Vie de saint Martin, II, 97-103 : "Hinc naturalis praecisi margine saxi/ambierat modico rupis curuata recessu, /hinc Liger inflexis paruo sinuamine ripis/ sepserat ingressam praetento gurgite terram./Unus et angustus patuit rupem inter et amnem /ingressus, paulum artato qua limite distans/fluminis allapsus tangit confinia montis. "

23. VIRGILE, Énéide, VIII, 233 : "praecisis undique saxis »-PAULIN DE PÉRIGUEUX, Vie de saint Martin, II, 97 : "praecisi... saxi ».

24. LABARRE, Sylvie, op. cit., p. 169-201. 
Paulin emploie aussi l'expression virgilienne auri fames qui rappelle l'appétit de l'or, responsable de la mort de Polydore (Énéide, III, 57), pour commenter l'interdiction faite aux moines de vendre quoi que ce soit : "À personne la possibilité de vendre quelque chose n'était concédée, de peur que ne s'accrût la faim détestable d'un or auquel on aurait goûté ${ }^{25}$. " Cette expression est devenue proverbiale dans la littérature latine, en prose comme en poésie, chez les païens (Pline l'Ancien et Quintilien) comme chez les chrétiens (Jérôme, Ambroise, Prudence, Sédulius) ${ }^{26}$. Cassien l'emploie comme Paulin de Périgueux, pour décrire l'appétit de l'or qui s'accroît chez le moine à proportion de ses gains ${ }^{27}$.

François Châtillon a relevé quelques parallèles textuels troublants entre Paulin de Périgueux et un autre poète, Sidoine Apollinaire, un évêque du même milieu, sensiblement de la même époque, puisqu'il naquit à Lyon, vers $431^{28}$. Ce sont surtout les poèmes II, V, et VII, c'est-à-dire les panégyriques d'Anthémius, de Majorien et d'Avitus, qui auraient été mis à contribution par l'auteur de la Vie de saint Martin. Des similitudes de vocabulaire existent entre la description du uicus Helena par Sidoine (Poèmes, V, v. 213-221) et la description de Marmoutier par Paulin (II, v. 84-155). Ce uicus Helena est le lieu d'un engagement entre Majorien et les Francs près d'Arras. Dans son panégyrique, Sidoine décrit des paysages fluviaux, des défilés et des collines. Châtillon estime qu'un poète hagiographe peut facilement s'approprier les expressions d'un panégyriste, car la description du lieu se présente de manière conventionnelle, avec des formules utilisables en plusieurs circonstances. Cependant il n'y a pas d'imitation manifeste, car on ne trouve aucune alliance de mots significative. La présence de uia, angustus ou flumen ne prouve rien. C'est seulement la description générale des lieux qui rapproche les deux poètes.

La réécriture poétique de Paulin est marquée par une constante amplification et une méditation spirituelle, si bien qu'il transpose le chapitre de la Vie de saint Martin consacré à Marmoutier, soit 34 lignes, chez Sulpice, en un long développement de soixante-douze vers (II, 84-155). Il explique ainsi le choix du bois pour la construction de la cellule de l'ascète : "C'est ici que Martin préféra se construire une petite cellule avec un amas de bois, car il connaissait les mystères du saint bois " (II, 104-105). Il établit un lien entre le matériau de construction et la Croix du Christ, selon une symbolique qu'on retrouve dans des poèmes de la même époque : le poème

25. PAulin De PÉRIgueux, Vie de saint Martin, II, 117-118 : "Nulli uendendi quicquam concessa facultas, /ne degustati fames improba cresceret auri. "

26. LABARRE, Sylvie, op. cit., p. 192; COURCELlE, Pierre, Lecteurs païens et lecteurs chrétiens de l'Énéide, t. 1, Paris, 1984, p. 230-234. Voir aussi art. "fames ", Thesaurus Linguae Latinae, vol. 6,1 , col. 233 .

27. CASSIEN, Institutions cénobitiques, VII, 7, 3 : "Auidior fames adcrescit auri tantoque uehementior suscitatur, quanto etiam summa lucri maior adponitur. "

28. ChÂtıllon, François, "Paulin de Périgueux, auteur de la Vita Martini et Sidoine Apollinaire, panégyriste des empereurs ", Revue du Moyen Âge Latin, t. 23, 1976, p. 5-12. 
pseudo-cyprianique De Pascha (uel de ligno uitae) ou l'Alethia de Claudius Marius Victorius (I, 546-547) ${ }^{29}$.

Le travail de copiste autorisé par Martin est ainsi justifié : « Il ne concède que le goût de l'écriture, parce que, pensait-il, elle occupe l'esprit, les yeux et la main, et retient sur un seul point toutes les facultés ensemble, les yeux par le regard, le cœur par la réflexion, par le tracé des lignes la main $^{30}$. " Il s'agit déjà d'une anthropologie médiévale dont l'origine remonte à Origène : le corps humain est unifié par la spiritualité. Cependant seuls les plus jeunes moines de Marmoutier sont occupés au scriptorium. C'est probablement parce qu'eux seuls n'étaient pas encore atteints de presbytie, mais Paulin trouve une autre explication. L'écriture est un service dû par les moines encore en formation, et il oppose les jeunes à ceux qui sont plus mûrs et auxquels la vie contemplative suffit : " ̊̀ ces travaux seul le jeune âge est occupé. Car à l'esprit déjà mûr la prière suffit pour que celui dont la sagesse plus âgée affermit la pensée se consacre à la pureté du cœur et veille à éclaircir l'œil intérieur, de peur que ce dernier, s'il était voilé par la nuée d'une faute cachée, ne soit privé de la lumière du Christ ${ }^{31}$. " L'œil intérieur représente l'œil du cœur, qu'évoque saint Paul (Éphésiens, 1, 18) et qui permet de voir les réalités spirituelles, mais cet œil doit être purifié, car le péché l'a aveuglé, selon notamment saint Augustin ${ }^{32}$.

Paulin ne se contente pas d'introduire un commentaire moral et spirituel, il donne aussi une version plus occidentale des Dialogues de Sulpice, et donc du monachisme. Selon la division carolingienne en 3 dialogues, le premier dialogue traite des moines d'Égypte, avant que Gallus ne prenne à son tour la parole pour montrer la supériorité des vertus morales et thaumaturgiques de Martin sur celles des moines d'Égypte. Il a complètement supprimé cette " matière d'Égypte ». Il n'a plus besoin de comparer Martin aux moines d'Orient.

Il présente également une version plus actuelle. Il met à jour le dossier martinien, en ajoutant un sixième chant consacré aux miracles posthumes de Martin, dont Perpetuus avait rédigé une première version en prose. Il y évoque les travaux de construction de la nouvelle basilique Saint-Martin, ordonnés par Perpetuus à Tours, les ravages des Huns, les combats des gallo-romains contre les Visigots.

29. Poème De Pascha (uel de ligno uitae), éd. HARTEL, CSEL, 3, 3, p. 305-308. On le date actuellement du v viècle; PRIEUR, Jean-Marc, La Croix dans la littérature chrétienne des premiers siècles, Bern, Berlin, Bruxelles, 2006, p. 226-227 (texte); La Croix chez les Pères (du It siècle au début du IV siècle), Strasbourg, 2006, p. 170-172 (commentaire).

30. PAulin DE PÉRIgueux, Vie de saint Martin, II, 119-122 : "Conceditur unum /scribendi studium, quod mentem oculosque manusque /occupet atque uno teneat simul omnia puncto, /aspectum uisu, cor sensibus, ordine dextram. "

31. PAulin DE PÉRIGUeux, Vie de saint Martin, II, 123-128 : "Attamen his rebus minor aetas sola tenetur. /Nam iam maturae satis est oratio menti, /ut, cuius sensum senior sententia firmat, /munditiis cordis studeat uigiletque remotum /interius purgare oculum, ne nube latentis /peccati obductus priuetur lumine Christi. "

32. Augustin, Homélies sur l'Évangile de saint Jean, 2, 16. Voir art. "Auge ", Reallexikon für Antike und Christentum, 1, 1950, col. 957-969. 
Sa réécriture est aussi plus tourangelle, notamment parce qu'il seconde Perpetuus dans sa politique qui consacre Tours comme la ville de Martin. Il réinterprète le paysage tourangeau en fonction d'une topographie martinienne. Luce Pietri l'a bien noté : "À la cité christianisée a fait place une cité chrétienne : l'espace urbain, depuis l'épiscopat de Perpetuus, s'organise en fonction de la géographie que dessinent les loca sancta martiniens [...]. À ses yeux (ceux de Paulin de Périgueux) le cours de la Loire, dont il célèbre la beauté à la traversée de Tours, est providentiellement adapté, dans son tracé, à la topographie des lieux saints de la cité qu'il côtoie et sépare $^{33}$. " Paulin exprime ainsi cette prédestination de la Loire à accueillir le saint : " Le fleuve nourricier atteste l'œuvre de la vertu merveilleuse de Martin : il touche les murs contigus de la ville et lèche les rochers du flot. Situé au milieu, il sépare la cellule (cellam) et le tombeau (sepulcrum) ${ }^{34}$." On songe à la valeur symbolique du Tibre chez Virgile et dans l'idéologie romaine. La Loire, qui coule entre l'ecclesia et le monasterium, rappelle le Tibre de l'Énéide, qui fait glisser ses eaux devant la tombe (tumulus) du jeune Marcellus, le neveu d'Auguste ${ }^{35}$, bien qu'il n'y ait aucun emprunt textuel.

\section{Avec Fortunat, le moine s'efface devant l'évêque et l'intercesseur}

Autour de 575, Venance Fortunat, venu d'Italie, compose une nouvelle version poétique de la Vie de saint Martin, d'environ 2200 vers. C'est l'époque où Grégoire de Tours, qui est son ami et son protecteur, poursuit la politique de Perpetuus et fait pleinement de Tours la ville de Martin. Monachus y est employé onze fois, le plus souvent pour désigner les moines qui entourent Martin, une seule fois à propos de Martin luimême. Monasterium s'y trouve cinq fois ${ }^{36}$. Le poète mentionne la fondation d'un ermitage à Ligugé, puis à Marmoutier. Le monastère apparaît ensuite comme le lieu d'où Martin s'élance pour aller accomplir un miracle : " chaque fois que Martin quittait le monastère pour courir à la ville " (Vie de saint Martin, IV, 158). Par deux fois, c'est le seuil du monastère qui est évoqué, en des formules très proches, seuil que ne peut franchir le démon (Vie de saint Martin, IV, 391 : " avant même qu'il eût atteint le seuil du saint monastère ", ante monasterii quam limina tangeret almi) ou que franchit Martin (Vie de saint Martin, IV, 483 : " avant de franchir le seuil de son ermitage ", ante monasterii sed quam bene limen adiret).

La fondation de Marmoutier est résumée en trois vers par Venance Fortunat: "Puis il se construit un ermitage (monasterium) sous une haute falaise (rupe sub alta), demeurant moine (monachus) dans les honneurs du

33. Pietri, Luce, op. cit., p. 429.

34. Paulin de PérIgueux, Vie de saint Martin, VI, 71-74 : « [...] fluuius testatur alumnus / mirandae uirtutis opus, qui moenibus urbis / iunctus contiguis adlambit saxa fluentis. Hic medius cellam discriminat atque sepulcrum."

35. VIRGILE, Énéide, VI, 873-874.

36. FORTUNAT, Vie de saint Martin, I, 158, 220; IV, 158, 391, 483. 
pontificat. En nombre y affluent les saints essaims (examina) de frères ${ }^{37}$. " La réécriture de Fortunat correspond à une stylisation épique. L'alliance de mots, rupe sub alta, se trouve chez des auteurs épiques comme Virgile, Lucain ou Stace ${ }^{38}$. La verticalité de la falaise, la résistance du moine, le nombre des moines soulignent la grandeur du héros de l'hagiographie. La verticalité exprime la transcendance. Dans la Bible, la montagne est en effet le lieu de la rencontre avec Dieu.

L'application de Martin à prier sans cesse, pour éloigner une épidémie, fait l'objet d'une réécriture originale, grâce à la médiation de l'épopée virgilienne. Les trois nuits sans étoiles (totidem sine sidere noctes) que passe Énée à naviguer deviennent sept nuits sous les étoiles (totidem sub sidere noctes) que Martin consacre à la prière : "Sept jours, oui, et autant de nuits, il veilla, prosterné sous la voûte étoilée, sans briser le jeûne, sans interrompre pendant la même durée ses prières ${ }^{39}$. " La navigation et la prière sont mises en parallèle. Mais l'endurance de Martin l'emporte sur celle d'Énée et, pendant que le héros troyen erre en mer, le saint sait où il va, tout entier tendu vers Dieu.

Cependant le poète Fortunat ne dit pas un mot de la communauté de Marmoutier, ni de sa règle de vie. Or, à la même époque, Grégoire de Tours atteste que Marmoutier a acquis un nouveau prestige, puisque le toponyme "Marmoutier ", signifiant " monasterium maius " apparaît à ce moment-là ${ }^{40}$. Les cellules sont à nouveau occupées par des moines placés sous l'autorité de l'abbé ${ }^{41}$.

C'est parce que Fortunat n'entend pas témoigner, dans sa réécriture épique, du développement du monachisme martinien. Il se concentre sur la figure de l'évêque et plus encore de l'intercesseur : « Je t'en prie, enveloppe ma faiblesse de ton voile sacré, écarte de moi le feu qui m'épouvante en m'abritant sous ton manteau (tua lacerna)" (IV, 611-612). Toutefois, dans un poème à caractère épigraphique, destiné à être gravé dans la cellula, c'est-à-dire le secretarium attenant à la cathédrale de Tours où Martin se recueillait avant les offices et où il vêtit un pauvre (Dial., II, 1), Fortunat insiste sur le caractère paradoxal de cet anachorète qui vit au milieu du peuple de ses fidèles : "Séjournant dans cette demeure, il y observait la

37. FoRTUNAT, Vie de saint Martin, I, 220-222 : "Inde monasterium sibi condit rupe sub alta,/uir monachus perstans in pontificatus honore./Plurima conueniunt ubi sancta examina fratrum."

38. VIRGILE, Bucoliques, I, 56; LuCAIN, IV, 601; VI, 641; STACE, Thébaïde, XI, 741.

39. VIRGILE, Énéide, III, 203-204 : "Tris adeo incertos caeca caligine soles / erramus pelago, totidem sine sidere noctes "; FORTUNAT, Vie de saint Martin, IV, 446 : " septem nempe dies, totidem sub sidere noctes".

40. GRÉGOIRE DE TOURS, Histore des Francs, X, 31, 3 : « [Martinus] In monasterio uero qui nunc maior dicitur basilicam in honore sanctorum apostolorum Petri et Pauli aedificauit "; Libri de uirtutibus sancti Martini, I, 2: "Cumque ad cellulam Maioris monasterii peruenissset..."

41. PIETRI, Luce, op. cit., p. 425. GRÉGOIRE DE TOURS, Libri de uirtutibus sancti Martini, III, 42. 
retraite de l'ermite, puissant anachorète au sein des peuples ${ }^{42}$. " C'est cette solitude intérieure qui lui ouvre les portes du ciel : "Martin, exilé sur la terre, citoyen du ciel, avait l'habitude de s'enfermer pour ouvrir les portes de l'éther ${ }^{43}$. " Le paradoxe est souligné par une série d'antithèses : terre/ ciel, exilé/citoyen, enfermer/ouvrir.

Faut-il reconnaître dans le silence relatif de Fortunat à propos de Marmoutier le signe que la postérité du monastère de Tours était, déjà à son époque, bien inférieure à celle du monastère de Lérins, fondé par Honorat entre 400 et 410 ? Dans le Carmen 5 du livre II, le poète souhaite que les moniales de Sainte-Croix continuent d'observer la règle pour les vierges de Césaire, moine de Lérins et évêque d'Arles, adoptée par Radegonde et inspirée de celle d'Augustin : "Qu'elles conservent [...] la sainte règle du pieux pontife Césaire qui, sortant du lot de Lérins, fut évêque à Arles et resta moine tout en étant la gloire de l'épiscopat " (v. 67-70). La tension entre monachisme et épiscopat, qui avait été vive à la fin du IV siècle, s'est estompée, si bien qu'à partir du $\mathrm{vI}^{\mathrm{e}}$ siècle, de nombreux moines accèdent à l'épiscopat selon une tendance qui ne fera que se confirmer par la suite ${ }^{44}$.

$\mathrm{Au} \mathrm{v}^{\mathrm{e}}$ siècle, Paulin de Périgueux inscrit les thèmes principaux du monachisme martinien dans une tradition poétique (celle de l'épopée virgilienne), dans une anthropologie pré-médiévale et une géographie symbolique (tourangelle et ligérienne). En revanche, au $\mathrm{VI}^{\mathrm{e}}$ siècle, c'est la figure épiscopale qui triomphe au détriment de celle du moine. Si les motifs essentiels de ce monachisme ont été diffusés par les œuvres en prose de Sulpice Sévère, en revanche il est difficile de dire quelle influence ont eu les versions poétiques sur le développement de ce monachisme. Quelle lecture faisait-on, au haut Moyen Âge, des œuvres poétiques de Paulin de Périgueux ou de Venance Fortunat? Alcuin, nommé en 796 abbé de SaintMartin de Tours, et auteur d'une Vie de saint Martin très abrégée, ou Odon ${ }^{45}$, originaire de Tours et abbé de Cluny de 927 à 942, se sont-ils nourris de la lecture de ces auteurs? Il est à peu près certain qu'ils disposaient de ces œuvres. V. von Büren a montré que la bibliothèque de Cluny était organisée " autour du saint patron de l'abbé Odon, Martin de Tours ${ }^{46}$ ", et le grand

42. Fortunat, Poèmes, I, V, 5-6 : "Aede sub hac habitans, heremi secreta tenebat, / per medios populos anachorita potens".

43. Ibidem, 3-4 : "Exul enim terris, caeli incola, saepe solebat / clausus Martinus hinc aperire polos."

44. Histoire des saints et de la sainteté chrétienne, t. 4, Les voies nouvelles de la sainteté, 605-814, sous la direction de Pierre Riché, Paris, 1986.

45. VIELBERG, Meinolf, Der Mönchsbischof von Tours im «Martinellus ». Zur Form des hagiographischen Dossiers und seines spätantiken Leitbilds, Berlin, 2006, p. 295-303 sur la réception du dossier martinien par Odon de Cluny, mais il n'y a pas d'éléments sur la réception des hagiographies poétiques.

46. Von BÜREN, Veronika, "Le grand catalogue de la bibliothèque de Cluny ", dans Le gouvernement d'Hugues de Semur à Cluny, Actes du colloque scientifique international, Cluny, septembre 1988, p. 245-263, citation p. 259. 
catalogue, qui daterait du xi ${ }^{\mathrm{e}}$ siècle, mentionne la présence des Vies poétiques de Paulin de Périgueux et de Venance Fortunat ${ }^{47}$.

\section{RÉSUMÉ}

La Vie de saint Martin et les Dialogues de Sulpice Sévère abordent par le biais du récit les thèmes essentiels de la vie monastique : séparation du monde, vie en communauté, ascèse, vêtement, prière, séparation des sexes et exaltation de la virginité. Ces œuvres en prose tracent le portrait d'un évêque-moine tourné vers l'action et pratiquant un idéal de vie mixte qui allie action et méditation, solitude et présence au monde. Paulin de Périgueux, dans sa Vie de saint Martin en vers, décrit précisément le site de Marmoutier. Il amplifie la description en imitant Virgile : il fait par exemple du locus horridus un lieu de prière pour le saint. Il insiste sur le travail des copistes qui unifie le corps humain par la spiritualité. À la différence de Sulpice Sévère il ne compare plus Martin aux moines d'Orient. Un siècle plus tard, le poète Venance Fortunat réduit la fondation de Marmoutier à un cliché épique et une antithèse.

\section{ABSTRACT}

The Life of saint Martin and the Dialogs written by Sulpicius Severus develop, through narration, the main themes of monastic life: separation from the world, communal life, asceticism, clothing, prayer, separation of the sexes and exaltation of virginity. These prose works shape the portrait of an action-oriented monk-bishop, who practices an ideal of a mixed life that combines action with contemplation, and solitude with presence in the world. Paulinus of Périgueux, in his metrical Life of St Martin, describes precisely the site of Marmoutier. He amplifies the description by imitating Vergil: for instance the locus horridus is made a place for the praying saint. He puts emphasis on the copyist's work, which unifies the human body through spirituality. In contrast to Sulpicius, he no longer compares Martin with oriental monks. One century later, the poet Venantius Fortunatus reduces the foundation of Marmoutier to an epic stereotype and an antithesis.

47. DeliSLe, Léopold, Le Cabinet des manuscrits de la bibliothèque nationale, t. 2, Paris, 1874, p. 458 sqq. Sur la diffusion de Paulin de Périgueux, voir LABARRE, Sylvie, " La transmission de Paulin de Périgueux : les principes d'une nouvelle édition ", Revue d'histoire des textes, n. s., t. VII, 2012, p. 117-157. 